\title{
A comparison of sessional ratings of perceived exertion to cardiovascular indices of exercise intensity during competition in elite field hockey players
}

\author{
Andrew Scott Perrotta1,2, Darren E.R. Warburton ${ }^{1,2,3}$ \\ 1 Cardiovascular Physiology and Rehabilitation Laboratory University of British Columbia, Vancouver, Canada; \\ 2 Experimental Medicine Program, Faculty of Medicine, University of British Columbia, Vancouver, Canada; ${ }^{3}$ School of \\ Kinesiology, Faculty of Education, University of British Columbia, Vancouver, Canada
}

\section{Summary}

Study aim: Recent evidence has revealed a reduction in the strength of correlation between ratings of perceived exertion and a heart rate (HR) derived training load in elite field hockey players during competition. These competitive periods involve sustained levels of cardiovascular performance coupled with considerable time performing above the anaerobic threshold. As such, the purpose of this investigation was to examine the magnitude of correlation between ratings of perceived exertion and time spent above threshold and two HR derived training loads.

Material and methods: Seventeen $(\mathrm{n}=17)$ international caliber female field hockey players competing as a national team were monitored over four matches during a seven-day competition period within the 2016 Olympic Cycle. Cardiovascular indices of exercise intensity were derived from HR dynamics and were quantified through estimating time spent above anaerobic threshold (LT2), the Edwards training load model $\left(\mathrm{TL}_{\mathrm{ED}}\right)$ and the Polar Training Load $\left(\mathrm{TL}_{\mathrm{POL}}\right)$. Sessional ratings of perceived exertion (sRPE) were recorded after each match.

Results: 64 samples were recorded for analysis. HR derived $\left(\mathrm{TL}_{\mathrm{ED}} \& \mathrm{TL}_{\mathrm{POL}}\right)$ and $\mathrm{sRPE}$ training loads remained comparable between matches. A large correlation $(p=0.01)$ was observed between sRPE and each heart rate derived training load $\left(\mathrm{TL}_{\mathrm{ED}} \&\right.$ $\left.\mathrm{TL}_{\mathrm{POL}}\right)$. An unremarkable relationship $(p=0.06)$ was revealed between time spent above LT2 and sRPE.

Conclusions: Our results demonstrate $\mathrm{HR}$ derived training loads $\left(\mathrm{TL}_{\mathrm{POL}} \& \mathrm{TL}_{\mathrm{ED}}\right)$ exhibit a stronger correlation with sRPE than time spent above LT2 in elite field hockey players during competition.

\section{Keywords: Athlete Monitoring - National Team - Team Sport - Cardiovascular Physiology - Training Load}

\section{Introduction}

Current evidence examining the physiological demands imposed on elite female field hockey players during competition has established the importance towards elevated and sustained levels of cardiovascular performance coupled with considerable time performing above the anaerobic threshold [16]. Such demands have elicited significant heart rate (HR) derived training loads, suggesting the examination of HR dynamics is a valuable approach for coaches and practitioners when monitoring elite field hockey players [20].

Integrative support staff within high performance team sport often utilize sessional ratings of perceived exertion
(sRPE) [9] when monitoring the internal training load due to its strong correlation with HR derived training loads $[14,15]$. However, recent evidence has demonstrated variance in the correlation between SRPE and HR derived training loads in elite field hockey players during specific phases of a mesocycle, with competition demonstrating a significant reduction [19]. This observation is further supported in elite female soccer players where similar reductions in the strength of correlation are experienced during competitive match play [2].

The amount of time spent near and above the anaerobic threshold has thought too significantly influence an athlete's SRPE $[7,10]$. However, a recent systematic review [12] has emphasised the current paucity of inquiry towards examining this relationship during competition in 
elite team sport with existing literature exclusively focusing on training sessions in soccer players [6].

As such, the purpose of this investigation was to examine the correlation between SRPE and time spent above the anaerobic threshold and two HR derived training loads during a competition period in elite female field hockey players. In conducting this investigation, we hypothesized that each HR derived training load would demonstrate a stronger correlation with sRPE when compared to time spent above the anaerobic threshold.

\section{Material and methods}

Seventeen $(n=17)$ international caliber female field hockey players (Table 1) competing as part of a national team were monitored during a competitive microcyle. All participants were outfield players and were selected by the coaching staff prior to competition based on their technical, tactical and physiological performance abilities.

Each athlete provided written informed consent to participate in the day-to-day heart rate monitoring activities that were regularly performed as members of the national field hockey team. Written informed consent was provided by the national field hockey organisation for access to all heart rate and sRPE data for analysis. The current investigation was approved for and followed the recommendations of the Clinical Research Ethics Review Board at the University of British Columbia consistent with the procedures outlined in the Declaration of Helsinki.

This investigation was a descriptive correlation study examining the relationship between sRPE and three cardiovascular indices of exercise intensity during a competitive period within the 2016 Olympic Cycle. Four international matches involving competition against a top 5 International Hockey Federation world ranked team was used to examine the strength of correlation between SRPE and time spent above anaerobic threshold (>LT2) and two HR derived training loads utilized in intermittent team sport. To enhance the reliability within the study design, the following procedures were employed. A single examiner was present for the entire competition period and was responsible for managing heart rate monitors and for the collection and recording of all sRPE and cardiovascular measurements. The competitive period examined was selected for its standardized environmental conditions in the effort to limit the influence of external variables when collecting sRPE scores [11] and HR dynamics during exercise [5].

\section{Competition schedule}

The competition period involved the selected players $(n=17)$ competing in four matches over a seven-day period with each test beginning at 18:00 (Fig. 1). Environmental conditions throughout the investigation were comparable. Participants followed their daily nutrition and hydration regimen as provided by the national team registered dietician over the competition period and were encouraged to standardize their food and beverage intake prior to arriving for each match. Consumption of fluid and sodium throughout the investigation was performed ad libitum. On-field playing time and substitution frequency for each player was determined by the head coach and was based on the needs of each match.

\section{Quantification of heart rate derived training loads}

Each participant was provided a dedicated POLAR Team $^{2}$ heart rate monitor (Polar, Electro, Oy, Kempele, Finland) equipped to sample at $1000 \mathrm{~Hz}$ and programmed to record at $1 \mathrm{sec}$ intervals. The HR monitors were provided to each participant 10 min prior to beginning a warmup and were collected immediately upon the completion of a cool down. The calculation of each HR derived training load was standardized to include the total length of the match consisting of two 35 min periods. Two HR derived training loads utilized within intermittent team sport for their utility to quantify HR dynamics were examined and are expressed using arbitrary units (AU). The first HR derived training load was proposed by Edwards $\left(\mathrm{TL}_{\mathrm{ED}}\right)$ [8] and was calculated by summating the product of the accumulated duration ( $\mathrm{min}$ ) within each of the five HR training zones by the coefficient relative to each zone (50-59\% of HRmax $=1,60-69 \%$ of HRmax $=2,70-79 \%$ of HRmax $=$ 3, 80-89\% of HRmax $=4,90-100 \%$ of HRmax $=5$ ). The second HR derived training load examined was the Polar Training Load $\left(\mathrm{TL}_{\mathrm{POL}}\right)$ [18]. This training load was calculated in real time using the Polar Team ${ }^{2}$ computer software program and was developed utilizing Banister's original training impulse (TRIMP) method $[3,4]$ that was customized with a recovery estimation model [18]. Maximum

Table 1. Participant Characteristics $(n=17)$

\begin{tabular}{ccccc}
\hline \multirow{2}{*}{ Age [y] } & Height $[\mathrm{cm}]$ & Weight $[\mathrm{kg}]$ & \multicolumn{2}{c}{ Maximal aerobic power } \\
\cline { 4 - 5 } & & & YoYo $[\mathrm{m}]$ & $\mathrm{VO}_{2 \mathrm{max}}\left[\mathrm{mL}^{2} \mathrm{~kg}^{-1} \cdot \mathrm{min}^{-1}\right]$ \\
\hline $22.0 \pm 2.1$ & $167.4 \pm 5.3$ & $62.5 \pm 5.5$ & $1837.1 \pm 337.8$ & $51.8 \pm 2.8$ \\
\hline
\end{tabular}


a)

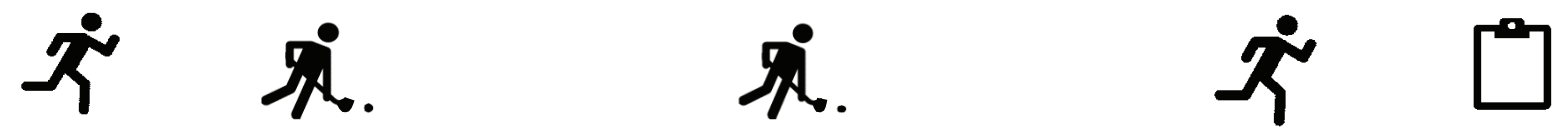

\begin{tabular}{|c|c|c|c|c|c|c|}
\hline \multicolumn{2}{|c|}{ Warm up (25 min) } & \multicolumn{3}{|c|}{ Competition (70 min) } & Cool down (20min) & RPE collection \\
\hline $\begin{array}{l}\text { Physiology oriented } \\
\text { (15min) }\end{array}$ & $\begin{array}{l}\text { Technical skills } \\
\text { oriented (10min) }\end{array}$ & $\begin{array}{l}\text { First half } \\
\text { (35 min) }\end{array}$ & $\begin{array}{l}\text { Half time } \\
\text { (10 min) }\end{array}$ & $\begin{array}{l}\text { Second half } \\
\text { (35 min) }\end{array}$ & Physiology oriented & Post cool down \\
\hline
\end{tabular}

b)

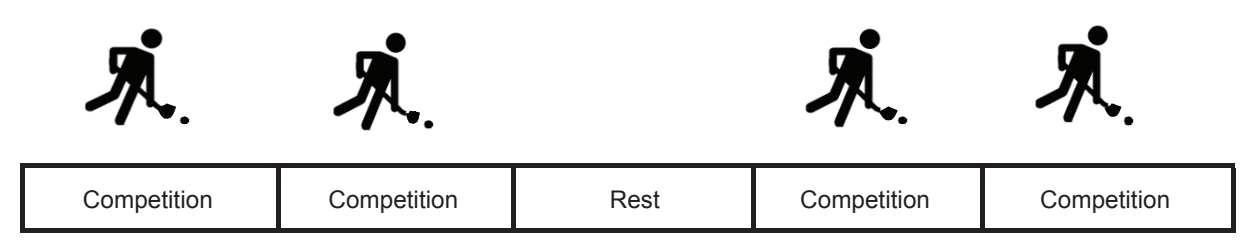

Fig. 1. a) format of individual match; b) format of competition period

HR values used to calculate each training load were determined from a graded maximal exercise test completed at the beginning of the mesocycle in which the current competition period was investigated. Heart rate monitors were collected after each match for exporting and calculating training loads into a Microsoft Excel database. If a participant's HR derived training load was not recorded for a match, the participants value was removed from analysis for that match

\section{Quantification for estimating time spent above anaerobic threshold (>LT2)}

Time ( $\mathrm{min}$ ) spent above the anaerobic threshold (>LT2) was estimated using each participants' HR value residing at lactate threshold two and was automatically calculated using the Polar Team ${ }^{2}$ computer software program. The HR value used to identify threshold was determined from a graded maximal exercise test completed at the beginning of the mesocycle in which the current competition period was investigated. An option within the computer software program was selected to use a standard deviation of beats per minute when identifying the time spent above threshold. This option was utilized to account for daily changes in plasma volume and fitness levels and was represented as a $3.0 \%$ coefficient of variation [5] around each participants' HR value at threshold.

\section{Quantification of perceived training loads (sRPE)}

A sessional rating of perceived exertion (sRPE) using a modified Borg Scale [9] (Table 2) was recorded 20 min after completion of a cool-down during each match. This time period was selected to allow for minimal interference with the national team's post-match routine, for its validity when performing light to vigorous exercise [23] and for accounting for any recency effect post match [9]. Training loads derived from sRPE scores were calculated through multiplying the total match length $(70 \mathrm{~min})$ by the sRPE score out of 10 [9]. All training load values are represented using arbitrary units (AU). If a participant's sRPE score was not recorded after a match, the participant's value was removed from analysis for that match.

\section{Statistical analysis}

All data in text, figures and tables are represented as mean $( \pm \mathrm{SD})$ unless otherwise stated. A Pearson Correlation coefficient $(r)(95 \% \mathrm{CL})$ analysis was utilized to examine the magnitude of correlation between SRPE and time spent above LT2 and between each HR derived training load. The following principles were applied for identifying the strength of correlation; $<0.10$ trivial, $0.10-0.29$ small, 0.30-0.49 moderate, 0.50-0.69 large, 0.70-0.89 very large and $0.90-1.0$ almost perfect [13]. A repeated

Table 2. Modified Borg Scale used to describe exercise intensity [9]

\begin{tabular}{lc}
\hline Rating & Descriptor \\
\hline 1 & Very, very easy \\
2 & Easy \\
4 & Somewhat Hard \\
5 & Hard \\
6 & \\
7 & Very hard \\
8 & \\
9 & \\
10 & Maximal \\
\hline
\end{tabular}


measures design was employed whereby all internal training loads and time spent above LT2 were compared over the competition period using a one-way, repeated measures ANOVA with a Bonferroni correction procedure for post hoc comparisons. Significance was declared using a probability of $p<0.05$.

\section{Results}

Throughout the competition period, 64 samples were recorded and included for analysis out of a possible 68 . Missing data sets were the result of players not selected for competition (3 samples) along with a HR monitor failing to record a training load value (1 sample). A Shapiro-Wilk analysis was used to examine the normality of each data set. An acceptable range in the kurtosis $(0.30,0.70)$ and skewness $(-0.70,-0.46)$ for each data set was observed. No substantial differences in SRPE or HR derived training loads were observed throughout competition (Table 3). Time spent above LT2 was notable lower in match\#1 when compared to other matches $(p=0.002)$. The strength of correlation between SRPE and each cardiovascular indices is accompanied with a 95\% CL and its corresponding magnitude in Table 4. A large correlation was observed between SRPE and each HR derived training load, $\mathrm{TL}_{\mathrm{POL}}$ $(r=0.63, p=0.01)$, and $\mathrm{TL}_{\mathrm{ED}}(r=0.60, p=0.01)$. An unremarkable association between sRPE and time spent above LT2 was identified $(r=0.46, p=0.06)$.

\section{Discussion}

The purpose of this investigation was to examine the correlation between SRPE and cardiovascular indices of exercise intensity during competitive match play to support practitioners when monitoring their athletes during critical periods of a yearly training plan. The findings presented in the current investigation suggest exercising HR dynamics when expressed as a training load $\left(\mathrm{TL}_{\mathrm{POL}}\right.$ or $\mathrm{TL}_{\mathrm{ED}}$ ) may better complement sRPE when monitoring exercise intensity during competition in elite field hockey players when compared to time spent above LT2.

Previous inquiry into female field hockey players has revealed increasing levels of anxiety in the lead up to competition [22]. This observation has been postulated to support the significant correlation observed between cognitive interference provoked from competition anxiety

Table 3. Comparison between cardiovascular and subjective indices of exercise intensity over four competitive matches $(\mathrm{n}=17)$

\begin{tabular}{lcccc}
\hline & Match \#1 & Match \#2 & Match \#3 & Match \#4 \\
\hline $\mathrm{TL}_{\text {POL }}$ & $167.9 \pm 27.8$ & $185.25 \pm 36.6$ & $179.6 \pm 40.5$ & $182.0 \pm 31.1$ \\
$\mathrm{TL}_{\mathrm{ED}}$ & $188.1 \pm 22.6$ & $196.7 \pm 30.9$ & $192.2 \pm 34.2$ & $192.6 \pm 27.5$ \\
$\mathrm{TL}_{\text {sRPE }}$ & $627.3 \pm 46.6$ & $625.1 \pm 41.8$ & $620.5 \pm 46.2$ & $628.3 \pm 62.7$ \\
$>\mathrm{LT}_{(\min )}$ & $26.6 \pm 7.0 *$ & $36.8 \pm 9.7$ & $36.1 \pm 12.0$ & $36.6 \pm 8.5$ \\
\%HR $_{(\max )}$ & $80.0 \pm 4.0$ & $79.0 \pm 6.0$ & $78.0 \pm 8.0$ & $79.0 \pm 5.0$ \\
\hline
\end{tabular}

Values represented as mean $( \pm \mathrm{SD})$. Training loads are expressed using arbitrary units $(\mathrm{AU})$. Abbreviations: Polar training load $\left(\mathrm{TL}_{\mathrm{POL}}\right)$ [18], Edwards training load $\left(\mathrm{TL}_{\mathrm{ED}}\right)$ [8], session rating of perceived exertion $\left(\mathrm{TL}_{\mathrm{sRPE}}\right)$ [9], time spent above anaerobic threshold $\left(>\mathrm{LT} 2_{\min }\right)$, and percentage of maximum heart rate $\left(\% \mathrm{HR}_{\max }\right)$. ${ }^{*}$ Denotes a substantial difference between matches $(p=0.002)$.

Table 4. Correlation between cardiovascular indices of exercise intensity and sRPE $(n=17)$

\begin{tabular}{lccc}
\hline & Total training load & $r(95 \%$ CL $)$ & Magnitude \\
\hline $\mathrm{TL}_{\text {POL }}$ & $662.0 \pm 147.9$ & $0.63(0.21,0.85) *$ & Large \\
$\mathrm{TL}_{\text {ED }}$ & $713.0 \pm 136.6$ & $0.60(0.17,0.84) *$ & Large \\
$\mathrm{TL}_{\text {sRPE }}$ & $2280.2 \pm 295.7$ & & Moderate \\
$>\mathrm{LT}_{(\min )}$ & $126.0 \pm 36.7$ & $0.46(-0.03,0.77)$ & \\
\hline
\end{tabular}

Values are represented as mean $( \pm \mathrm{SD})$. A Pearson Correlation coefficient $(r)(95 \% \mathrm{CL})$ is provided between sRPE and each heart rate derived training load $\left(\mathrm{TL}_{\mathrm{POL}} \& \mathrm{TL}_{\mathrm{ED}}\right)$ along with time spent above threshold ( $\left.>\mathrm{LT} 2\right)$. Correlation coefficients are accompanied with their respective magnitude. ${ }^{*}$ Denotes the strength of correlation possessing a $p<0.05$ 
and on-field performance in elite field hockey players during international competition [21]. The concomitant surge in cortisol from the inception of competition anxiety has demonstrated to be significantly correlated with SRPE, further supporting its validity and utility for monitoring the perceived exercise stress during competition [17]. A limitation in the current investigation was the absence of a psychological assessment tool or the examination of serum levels of stress hormones that would have permitted comparisons between each cardiovascular indices of exercise intensity. However, based on the current findings, we postulate the large and remarkable correlation between each HR derived training load $\left(\mathrm{TL}_{\mathrm{POL}} \& \mathrm{TL}_{\mathrm{ED}}\right)$ and $\mathrm{SRPE}$ suggest time spent above LT2 may not sufficiently demonstrate the psychophysiological element that accompanies competitive match in elite field hockey players throughout competition.

The utility of HR monitoring equipment that offers in real time analysis and a programmed training load calculation allows practitioners to efficiently monitor and quantify exercise intensity throughout competitive match play. This option may enhance the decision-making process towards individualizing post-match recovery procedures and designing future training sessions prior to forthcoming competition. Each HR derived training load in the current study demonstrated a large and substantial correlation with sRPE, however, the $\mathrm{TL}_{\mathrm{POL}}$ model provided an immediate calculation whereas the $\mathrm{TL}_{\mathrm{ED}}$ model required manual, retrospective calculation. A previous review focusing on sport practitioners within professional soccer clubs has suggested that man power is the critical limitation in both data acquisition and its analysis [1]. This evidence is compelling for implementing effective yet efficient monitoring techniques that allow practitioners within team sport to make evidence-based decisions from valid and immediate data acquisition. As such, the utilization of a valid HR derived training load, that can be calculated in real time, may be a critical component for practitioners when monitoring performance and recovery status during competitive periods that demand sustained cardiovascular performance.

\section{Conclusions}

In summary, this investigation identified a large and remarkable correlation between sRPE and each HR derived training load $\left(\mathrm{TL}_{\mathrm{POL}} \& \mathrm{TL}_{\mathrm{ED}}\right)$ when compared to time spent above LT2. These findings encourage practitioners to focus on HR derived training loads in combination with SRPE when examining both objective and subjective markers of exercise intensity during competition. Moreover, the use of exercise equipment that can analyze HR dynamics and calculate training loads in real time, may further support coaches and practitioners to make evidence-based decisions using immediate analysis to select recovery techniques and plan for imminent training sessions within a competition schedule.

\section{Conflict of interest: Authors state no conflict of interest.}

\section{References}

1. Akenhead R., Nassis G.P. (2016) Training Load and Player Monitoring in High-Level Football: Current Practice and Perceptions. Int. J. Sports Physiol. Perform., 11: 587-593.

2. Alexiou H. Coutts A.J. (2008) A comparison of methods used for quantifying internal training load in women soccer players. Int. J. Sports Physiol. Perform., 3: 320.

3. Banister E. (1991) Modeling elite athletic performance. Physiological Testing of Elite Athletes, 403-424.

4. Banister E., Morton R., Fitz-Clarke J. (1992) Dose/response effects of exercise modeled from training: physical and biochemical measures. Ann. Physiol. Anthropol., - Seiri Jinruigaku Kenkyūkai Kaishi 11: 345.

5. Buchheit M. (2014) Monitoring training status with HR measures: do all roads lead to Rome? Front. Physiol., 5: 73 .

6. Campos-Vazquez M.A., Mendez-Villanueva A., Gonzalez-Jurado J.A., Leon-Prados J.A., Santalla A., SuarezArrones L. (2015) Relationships Between Rating of Perceived Exertion and Heart-Rate-Derived Internal Training Load in Professional Soccer Players: A Comparison of On-Field Integrated Training Sessions. Int. J. Sports Physiol. Perform., 10: 587-592.

7. Carey D.L., Ong K., Morris M.E., Crow J., Crossley K.M. (2016) Predicting ratings of perceived exertion in Australian football players: methods for live estimation. Int. J. Comput. Sci. Sport, 15: 64-77.

8. Edwards S. (1993) High Performance Training and Racing. In: The Heart Rate Monitoring Book, (eds.): Edwards S. (Sacramento, CA:Feet Fleet Press) p. 113-123.

9. Foster C., Florhaug J.A., Franklin J., Gottschall L., Hrovatin L.A., Parker S., Doleshal P., Dodge C. (2001) A new approach to monitoring exercise training. J. Strength Cond. Res., 15: 109-115.

10. Haddad M., Chaouachi A., Wong D.P., Castagna C., Hue O., Impellizzeri F.M., Chamari K. (2014) Influence of exercise intensity and duration on perceived exertion in adolescent Taekwondo athletes. Eur. J. Sport Sci., 14 Suppl 1: S275-S281.

11. Haddad M., Padulo J., Chamari K., (2014) Sport Science Program CoAaSQUDQ. The Usefulness of Session Rating of Perceived Exertion for Monitoring Training Load Despite Several Influences on Perceived Exertion. Int. J. Sports Physiol. Perform., 9: 882-883. 
12. Haddad M., Stylianides G., Djaoui L., Dellal A., Chamari K. (2017) Session-RPE Method for Training Load Monitoring: Validity, Ecological Usefulness, and Influencing Factors. Front. Neurosci., 11: 612.

13. Hopkins W., Marshall S., Batterham A., Hanin J. (2009) Progressive statistics for studies in sports medicine and exercise science. Med. Sci. Sports Exerc., 41: 3.

14. Impellizzeri F.M., Rampinini E., Coutts A.J., Sassi A., Marcora S.M. (2004) Use of RPE-based training load in soccer. Med. Sci. Sports Exerc., 36: 1042-1047.

15. Kelly D.M., Strudwick A.J., Atkinson G., Drust B., Gregson W. (2016) The within-participant correlation between perception of effort and heart rate-based estimations of training load in elite soccer players. J. Sports Sci., 34: 1328-1332.

16. McGuinness A., Malone S., Hughes B., Collins K. (2018) The Physical Activity and Physiological Profiles of Elite International Female Field Hockey Players Across the Quarters of Competitive Match-Play. J. Strength Cond. Res., DOI: 10.1519/JSC.0000000000002483. Published ahead of print.

17. Moreira A., McGuigan M.R., Arruda A.F.S., Freitas C.G., Aoki M.S. (2012) Monitoring Internal Load Parameters During Simulated and Official Basketball Matches. J. Strength Cond. Res., 26: 861-866.

18. Nissilä J., Kinnunen H. (2008) Heart rate based training load and recovery time estimation. Polar Electro $O y, 1: 1-4$.

19. Perrotta A.S., Held N.J., Warburton D.E.R. (2017) Examination of internal training load parameters during the selection, preparation and competition phases of a mesocycle in elite field hockey players. Int. J. Perform. Anal. Sport, 17: 813-821.
20. Perrotta A.S., Taunton J.E., Koehle M.S., White M.D., Warburton D.E.R. (2018) Monitoring the Prescribed and Experienced Heart Rate Derived Training Loads in Elite Field Hockey Players. J. Strength Cond. Res., DOI: 10.1519/JSC.0000000000002474. Published ahead of print.

21. Pérez-Encinas C., Fernández-Campos F.J., Rodas G., Barrios C. (2016) Influence of Cognitive Interferences and Self-Talk Functions on Performance During Competition in Elite Female Field Hockey Players. J. Strength Cond. Res., 30: 3339-3346.

22. Thatcher J., Thatcher R., Dorling D. (2004) Gender differences in the pre-competition temporal patterning of anxiety and hormonal responses. J. Sports Med. Phys. Fitness, 44: 300-308.

23. Uchida M.C., Teixeira L.F.M., Godoi V.J., Marchetti P.H., Conte M., Coutts A.J., Bacurau R.F.P. (2014) Does the Timing of Measurement Alter Session-RPE in Boxers? J, Sports Sci. Med., 13: 59-65.

\section{Received 03.07.2018 \\ Accepted 29.10.2018}

(c) University of Physical Education, Warsaw, Poland

Acknowledgments

This investigation was funded through the Natural Sciences and Engineering Research Council of Canada (NSERC) 